\title{
Construction of the Optimization Model of Cargo Transport Network
}

\author{
Kong Yanyan ${ }^{1, a^{*}}$, Tian Benfang ${ }^{1, b}$, Wang Qingyang ${ }^{2, c}$,Liu Donghui ${ }^{1}$,Gao Yunfei ${ }^{2}$ Li Chuang $^{2}$ and Zhang Yiwen ${ }^{3}$ \\ ${ }^{1}$ Logistic engineering Department, Shandong Jiaotong University, Jinan Shandong Province, 250357, China \\ ${ }^{2}$ Traffic engineering Department, Shandong Jiaotong University, Jinan Shandong Province, 250357, China \\ ${ }^{3}$ Traffic equipment and control engineering Department, Shandong Jiaotong University, Jinan Shandong Province, 250357, China
}

\begin{abstract}
The transportation is gradually integrated into the circulation system of bulk goods, and developing and growing. Transportation has the advantages of high efficiency, high quality and green environment, In time, efficiency and cost than the traditional bulk transport has certain advantages. This paper analyzes the cost of logistics transportation network, which mainly includes transportation cost in transit, transit cost, time cost of cargo transportation and special cost. This paper discusses in detail the transportation cost, transit cost, time cost and carbon consumption cost of different transportation modes in the process of "scattered transformation", and constructs the optimization model of" scattered transformation "transportation network with the least comprehensive transportation cost including the above costs.
\end{abstract}

\section{Introduction}

Low carbon is closely related to transportation. As an important part of the transportation industry, bulk cargo transportation has formed the four modes of bulk transportation, bulk transportation, bulk unloading and bulk storage for a long time, which pollutes the port and urban environment to a large extent. As a green and efficient mode of transportation, container transportation has developed rapidly. In addition, it can reduce the trade imbalance of container liner shipping lines, effectively reduce the transportation cost of cargo owners, and thus reduce the logistics cost of the whole society. Based on this background, it is particularly important to discuss and study the transportation problem of "changing scattered to centralized".

The transportation of "loose change" is beneficial to reduce the trade imbalance of container liner route, and can effectively reduce the transportation cost of cargo owners, thus reducing the logistics cost of the whole society. Transportation can meet the requirements of small batch, multi-variety and different quality grade, and can use various means of transportation of railway, waterway and highway to meet different time requirements, so that multimodal transport can be further developed. Multimodal transport can shorten the transportation time, reduce the transportation cost to a large extent, reduce the road congestion, improve the quality of transportation, and the environmental protection benefit is remarkable, which can not only achieve the improvement of energy efficiency, but also reduce the noise pollution and $\mathrm{CO} 2$ emissions.

Some enterprises that carry out "scattered" transportation only pay attention to the costs directly

* Corresponding author: ${ }^{\mathrm{a} 1652214425 @ q q . c o m}$

b3266156130@qq.com

c1181359962@qq.com related to transportation. In network optimization, sometimes they do not consider the carbon costs brought by carbon tax collection, which leads to the lack of rationalization of transport networks. Carbon emissions can not be reduced effectively. Transportation is different from bulk transportation. The optimization of transportation network technology needs to consider multiple nodes and transportation modes. The conversion speed between different transportation modes directly affects the overall transportation efficiency. How to optimize the transportation network is very important.

\section{Analysis on Optimization of Cargo Transport Network}

Suppose there are multiple trans-shipment points Distribution Network between the $\mathrm{O}$ of the starting point and the destination $\mathrm{D}$, in the actual transport network, these nodes represent the form of road container transit stations, container ports, railway container handling stations and air ports. s modes of transport are available between the connected nodes, with different modes of transport having different carbon costs, transport costs and time, Here we do not consider the multi-way distribution of goods, and any mode of transport between the two nodes has a corresponding determination of the transport path, and the node allows the transfer of goods, in the event of transit needs a certain transit time, resulting in a certain transfer cost. Under the constraint of not exceeding the transportation time limit, the enterprise is required to select the network node that needs the way, and at the same time, it is necessary to decide which transportation mode to select for each transportation section, so as to minimize 
the comprehensive transportation cost of the enterprise. The comprehensive transportation cost includes carbon cost, in-transit transportation cost, transit cost, cargo transportation time cost and special cost. The construction of transport network map is indispensable in the research of low-carbon transport network optimization. the network is described by a directed graph $\mathrm{G}(\mathrm{a}, \mathrm{N})$ according to graph theory, in which the $\mathrm{N}$ is the collection of each node of the "scattered transformation set" freight network, which is the main gathering place, transit place and receiving place; $\mathrm{A}$ is the arc set of the transportation line in the network diagram, the transportation line is the flow channel of the "scattered" goods produced by the network node, and the "scattered" box quantity in the transportation route is the capacity of the arc in the diagram. The transport network diagram is shown in figure 1 .

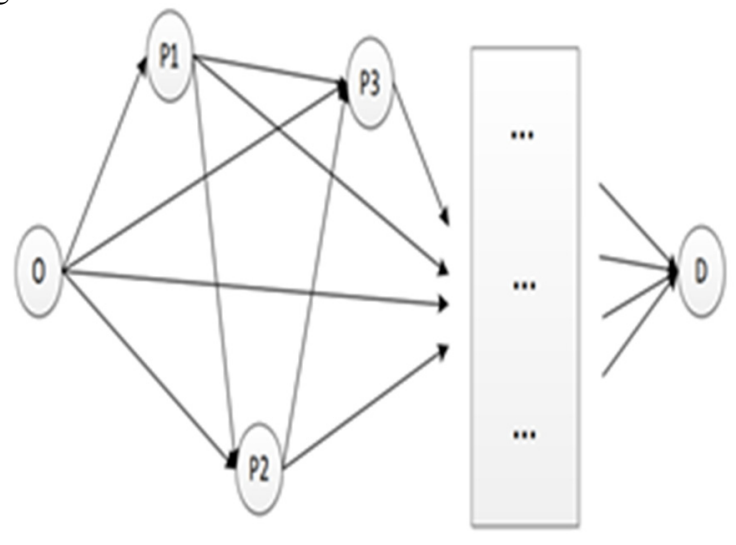

Fig. 1. Caption of the Figure 1. Below the figure.

If the transport mode conversion is carried out at a node, the conversion process is shown in figure 2 . The solid frame represents a transport node, each node can choose three modes of transport, namely, road, railway, waterway.

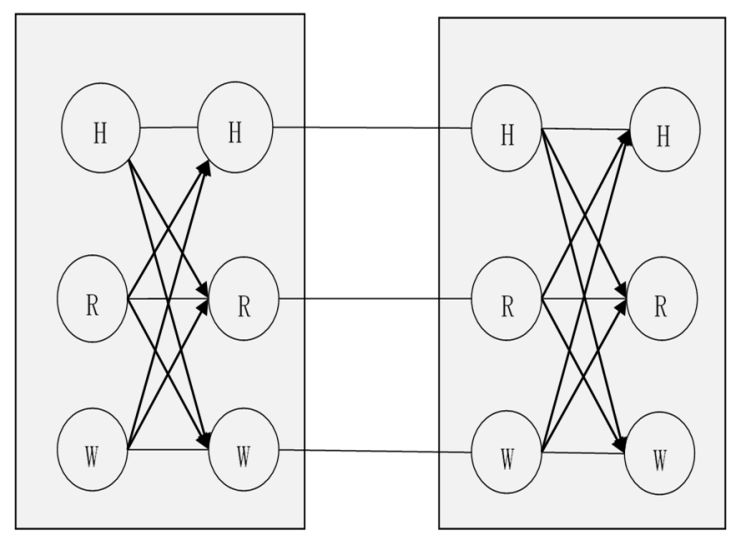

Fig. 2. Transport Node Transhipment Chart.

If we need to describe the change of transportation mode at a certain node in detail, we can combine figure 1 and figure 2 to establish another commonly used extended network diagram as shown in figure 3 . The transport network diagram extends the urban node according to the type of mode available at the time of arrival. A virtual final destination D1. is needed to ensure the integrity of the network diagram, In order to simplify the network diagram, we can set up virtual nodes to represent direct transportation. A point represents the transit node, the B point represents the virtual node, and the direct transportation through the virtual node B is called crosslevel transportation. The network diagram can directly reflect the transit time, transit carbon emissions and transit costs of goods, one side represents a mode of transport.

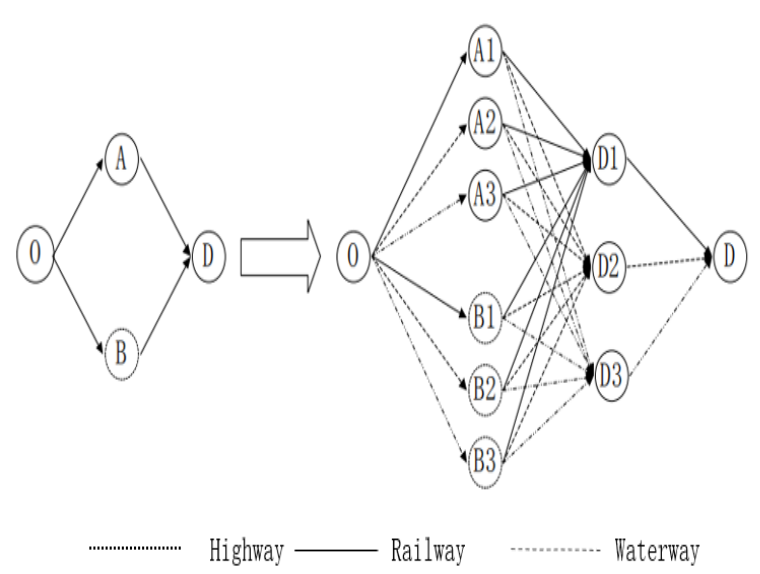

Fig. 3. Extended Network Diagram

\section{Optimization Model of Cargo Transport Network}

The transportation cost includes carbon cost, transportation cost, transit cost, transportation time cost and special cost. Taking the sum of the above cost as the objective function, the optimization model of low-carbon transport network for multimodal transport of goods is obtained:

$$
\begin{gathered}
\min Z=C_{e}+C_{T}+C_{V}+C_{I} \\
\min Z=c_{e} \sum_{k, m=1}^{S} \sum_{i, j=1}^{N}\left(\lambda * Q * D_{i j}^{k} * U C_{k} * x_{i j}^{k}+\lambda *\right. \\
\left.Q * U Z_{k m} * y_{i}^{k m}\right)+\sum_{k, m=1}^{S} \sum_{i, j=1}^{N}\left(C_{i j}^{k} * x_{i j}^{k}+\lambda * Q *\right. \\
\left.R E_{i}^{k m} * y_{i}^{k m}\right)+\sum_{k, m=1}^{S} \sum_{i, j=1}^{N}\left(C_{i j}^{k} * x_{i j}^{k}+\lambda * Q *\right. \\
R E_{i}^{k m} * y_{i}^{k m}+\lambda * Q *\left(Y_{O}+Y_{D}\right)
\end{gathered}
$$

The constraints are:

$$
\sum_{i, j} \sum_{k} T_{i j}^{k} * x_{i j}^{k}+\sum_{i} \sum_{k, m \in N} t_{i}^{k m} y_{i}^{k m} \leq T \quad i, j \in
$$

$N ; k, m \in S$

$$
\begin{array}{cc}
\sum_{k} x_{i, i+1}^{k}-\sum_{k} x_{i-1, i}^{k}=1 & i=0, k \in S \\
\sum_{k} x_{i, i+1}^{k}-\sum_{k} x_{i-1, i}^{k}=0 & i \in I, k \in S \\
\sum_{k} x_{i, i+1}^{k}-\sum_{k} x_{i-1, i}^{k}=-1 & i=D, k \in S \\
x_{i j}^{k} * x_{j h}^{m}=y_{j}^{k m} & i, j, h \in N ; k, m \in S \\
\sum_{i \in N} \sum_{k \in S} x_{i, i+1}^{k}=1 & i=1,2 \ldots, N-1 \\
\sum_{k, m \in N} y_{i}^{k m} \leq 1 & i \in N \\
\sum_{i} \sum_{k, m \in N} y_{i}^{k m} \leq G & i \in N ; k, m \in S \\
x_{i j}^{k}, y_{i}^{k m} \in\{0,1\} & i, j \in N, k, m \in S
\end{array}
$$


Formula (1) to (2) represents the optimal function of integrated transportation cost, The Ce is carbon cost, the $\mathrm{CT}$ is the sum of transportation cost and transit cost, the $\mathrm{CV}$ is the time cost of goods transportation, and the $\mathrm{CI}$ is the special cost. Constraint (3) indicates that the total time of shipment (including transportation time and replacement time) does not exceed the time required by the customer. Constraints (4) to (6) guarantee a complete path from the starting point to the end. Constraints (7) ensure the continuity of the whole course of cargo transportation. Constraint (8) indicates that only one mode of transport is allowed between two transport nodes, that is, the volume can not be divided. Constraint (9) indicates that if transit occurs at a node, only one mode of transport is allowed to be converted to another. Constraint (10) indicates that the number of full transshipment of "bulk transfer" does not exceed G. maximum number of transshipment. In constraint condition (11), 1 means that the transport mode is adopted between the node and the node, otherwise it is $0 ; 1$ means that the node changes from the mode of transport to the mode of transport, otherwise it is 0 .

\section{Cost analysis of logistics transportation network}

\subsection{Cost of transportation in transit}

\section{(1) Transportation cost of road transportation.}

Because "bulk transfer" transportation is to transport bulk goods into containers, the transportation cost of "bulk transfer" can be calculated by reference to container transportation cost. Container road freight can be divided into short distance transportation and long distance transportation according to the length of transportation distance. The short distance freight rate is calculated according to the principle of long distance decline, while the long distance freight rate is calculated according to the basic freight rate. The "scattered change" freight transportation discussed in this paper refers to long distance transportation. The container road transportation uses the unified basic freight rate in our country, of which $20 \mathrm{ft}$ standard box is 6.00 yuan / box kilometer $40 \mathrm{ft}$ standard box is 9.00 yuan / box kilometer, allowing provinces and municipalities to make the basic freight rate of container road transportation in this area according to the local actual situation. The node $i$ to the node can be obtained according to the calculation method of the actual container road freight combined with the characteristics of the "scattered change set" cargo transportation $\mathrm{j}$ and the road transportation cost calculation method is shown in the following formula (here $\mathrm{k}=1$, for road transportation):

$$
C_{i j}^{k}=\lambda * B S_{h} * D_{i j}^{k}
$$

BSh means the basic freight rate for road transportation, yuan / TEU km;

(2) Transportation cost of "scattered reform" railway.

At present, domestic container railway tariff charging methods include dry rate and average rate. The Rules on Freight Rates of Railway Goods stipulate that the basic freight rates of container goods shall be calculated on the basis of the rates of container freight and the number of boxes used as stipulated in the Schedule of Freight Rates of Railway Goods. Besides the basic freight, the freight cost of container railway also includes the surcharge of railway electrification, the equal share of new road and the miscellaneous expenses of railway freight. The above cost can be converted into the basic freight $\mathrm{BSr}$. of railway transportation according to the actual transportation

The node can be $\mathrm{i}$ to the node according to the actual calculation method of container railway freight combined with the characteristics of "scattered collection" cargo transportation $\mathrm{j}$ and the railway transportation cost calculation method is shown in the following formula (here $\mathrm{k}=2$, for railway transportation)

$$
C_{i j}^{k}=\lambda * Q * B S_{r} * D_{i j}^{k}
$$

BSr means the basic freight rate, yuan / TEU km; for railway transportation

(3) At present, the shipping cost of container waterway mainly includes package rate, discount rate and uniform rate.

Container transportation mostly adopts liner transportation, because liner transportation generally uses dry rate, so this paper adopts container dry rate, dry rate varies with route. In addition, considering the imbalance of container route cargo flow, in the return container ship with high no-load rate, according to the actual situation, the use of "scattered transfer" transport bulk goods can be given a certain freight discount. The node can be $\mathrm{i}$ to the node according to the actual calculation method of waterway freight $\mathrm{j}$ and the waterway transportation cost calculation method is shown in the following formula (here $\mathrm{k}=3$ represents waterway transportation):

$$
C_{i j}^{k}=\lambda * Q * E_{i j}^{k} * \alpha
$$

The $\mathrm{E}$ indicates that the node $\mathrm{i}$ to the node $\mathrm{j}$ adopts the single box freight rate $\mathrm{k}$ by the mode of transportation. Here $\mathrm{k}=3$ represents the waterway transportation, and the yuan / TEU; $\alpha$ is the discount of the freight rate, $\%$;

\subsection{Transfer Cost of Bulk Transfer}

Transportation includes many kinds of transportation modes, which involve the transformation of transportation mode. The reasonable selection of nodes for the conversion of transportation mode and the transfer of goods to another mode of transportation at the node can effectively reduce the comprehensive cost of the whole process. The main function of the transfer node is to realize the transfer of goods by road, railway and waterway, which will produce the transfer cost. In order to simplify the calculation and more intuitively show the functional relationship between freight volume and transit cost, the method of calculating transit cost in this paper is as follows:

$$
C_{z i}=\lambda * Q * R E_{i}^{k m}
$$

The cost of transportation mode is equal to the sum of the transportation cost in transit between nodes and the transit cost at nodes, that is: 


$$
C_{T}=\sum_{k, m=1}^{S} \sum_{i, j=1}^{N}\left(C_{i j}^{k} * x_{i j}^{k}+\lambda * Q * R E_{i}^{k m} * y_{i}^{k m}\right)
$$

\subsection{Time cost of transportation of "bulk" goods}

The time cost of transporting goods includes the time value of the capital of the goods, which is related to the opportunity cost of the funds occupied by the goods in transit, it also includes reducing the loss of goods, market price changes, packaging costs, storage costs and other capital values. For the time cost of transportation of goods, it is generally related to the mode or route of transportation, the distance of transportation, the type of goods, the transportation interval or direction, and so on. When evaluating the time cost of cargo transportation, the above factors should be quantified. Therefore, the calculation formula of transportation time cost of goods is as follows:

$$
C_{V}=\sum_{i, j=1}^{N} \sum_{k, m=1}^{S} \lambda * Q * T V *\left(T_{i j}^{k} * x_{i j}^{k}+t_{i}^{k m} y_{i}^{k m}\right.
$$

From the point of view of the time value of transportation and the interest on the funds during the period, the cost coefficient of the time of transportation of the goods is calculated. The formula TV the time cost coefficient of the goods is as follows:

$$
T V=\frac{P_{r} * I R}{365 * 24}
$$

\subsection{Special Cost of Scattered Reform}

The transportation of bulk goods will be carried in containers, so there will be a certain packing fee and transfer fee $\mathrm{O}$ the starting point. The one-way heavy box transportation is considered here, so the return fee will not be considered. At the end D there will be a certain cost of taking out and cleaning. The cost is related to the quantity of the box and can be calculated by the following formula:

$$
C_{I}=\lambda * Q *\left(Y_{O}+Y_{D}\right)
$$

The YO indicates the packing fee and the transfer fee $\mathrm{O}$ the starting point, Yuan / box, and the YD means the cost of taking out and cleaning the terminal D, Yuan / box.

\section{Conclusion}

The optimization scheme of transportation network considering time limit and carbon emission is better. In this paper, cross-node transport is considered compared with most of the current transport network optimization models, in the future, we can study the network optimization problem considering that the transportation of goods between two nodes is shared by two or more modes of transportation.

\section{References}

1. Miao Hongyun. A Study on Optimization of Lowcarbon Transport Network for Goods [D].] Wuhan University of Technology, 2018
2. Miao Linzhan. Discussion on the Design of a New Type of Loading and Unloading System of "Dispersion and Reforming Collection "[ J].] Hoisting transport machinery. 2020(19)

3. Zhao Duofa. Equipment [J].] for port's "loose assembly" unpacking operation. Containerization. 2019 (02)

4. Xie Shouhong, Cai Haiya, Xia Gangxiang. Calculation and Influencing Factors of Carbon Emission in China Transportation Industry [J].] 1Resources and environment in arid areas. 2016(05).

5. Sun Jiaqing. A Some Thoughts on Coal Transportation under the Concept of Green Logistics [J].] of "Dispersion and Reform Coal China, 2013 (09):13-15

6. Duan Xueyan, Fan Chen. Optimization of Container Transport Network of Xiaonehe River in Yangtze River Delta under Low Carbon Economy [J].] China Navigation ,2016(01):110-114

7. Zhang Junwei. A Study on Route Rationalization of Full Container Transport Network in Multimodal Transport [D].] Beijing Jiaotong University ,2011. 